西

\title{
Up-Regulation of P21 Inhibits TRAIL- Mediated Extrinsic Apoptosis, Contributing Resistance to SAHA in Acute Myeloid Leukemia Cells
}

\author{
Xing Wu $u^{a, b, c} \quad N a$ Yang ${ }^{a, b} \quad$ Wei-hua Zhou ${ }^{d}$ Jie Xu ${ }^{d}$ Jia-jie Chen ${ }^{a}$ Fei-meng Zheng ${ }^{b}$ \\ Zi-jie Long ${ }^{a}$ Cai-feng Yue ${ }^{b}$ Ke-xin Ai ${ }^{a}$ Ling-ling Liu ${ }^{a}$ Xian-yao Wane Quentin Liu,a \\ aDepartment of Hematology, the Third Affiliated Hospital, Sun Yat-sen University, Guangzhou; Institute \\ of Cancer Stem Cell, Dalian Medical University, Dalian, 'bState Key Laboratory of Oncology in South \\ China, Cancer Center, Sun Yat-sen University, Guangzhou, 'Department of Oncology, the Third Affiliated \\ Hospital, Southern Medical University, Guangzhou, dDepartment of Oncology, the Second Affiliated

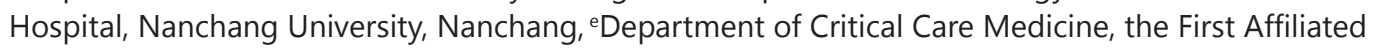 \\ Hospital, Dalian Medical University, Dalian, China
}

\section{Key Words}

P21 • SAHA • TRAIL • Histone deacetylase inhibitors • Acute myeloid leukemia

\begin{abstract}
Background/Aim: P21, a multifunctional cell cycle-regulatory molecule, regulates apoptotic cell death. In this study we examined the effect of altered p21 expression on the sensitivity of acute myeloid leukemia cells in response to HDAC inhibitor SAHA treatment and investigated the underlying mechanism. Methods: Stably transfected HL60 cell lines were established in RPMI-1640 with supplementation of G-418. Cell viability was measured by MTT assay. Western blot was applied to assess the protein expression levels of target genes. Cell apoptosis was monitored by AnnexinV-PE/7AAD assay. Results: We showed HL60 cells that that didn't upregulate p21 expression were more sensitive to SAHA-mediated apoptosis than NB4 and U937 cells that had increased p21 level. Enforced expression of p21 in HL60 cells reduced sensitivity to SAHA and blocked TRAIL-mediated apoptosis. Conversely, p21 silencing in NB4 cells enhanced SAHA-mediated apoptosis and lethality. Finally, we found that combined treatment with SAHA and rapamycin down-regulated p21 and enhanced apoptosis in AML cells. Conclusion: We conclude that up-regulated p21 expression mediates resistance to SAHA via inhibition of TRAIL apoptotic pathway. P21 may serve as a candidate biomarker to predict responsiveness or resistance to SAHA-based therapy in AML patients. In addition, rapamycin may be an effective agent to override p21-mediated resistance to SAHA in AML patients.
\end{abstract}

X. Wu, N. Yang and W.-h. Zhou contributed equally to this work.

Quentin Liu

and Xian-yao Wan
Department of Hematology, the Third Affiliated Hospital, Sun Yat-sen University, Guangzhou 510630, China; Institute of Cancer Stem Cell, Dalian Medical University, Dalian 116044, (China) and Department of Critical Care Medicine, The First Affiliated Hospital, Dalian Medical University, Dalian (China)

E-Mail liuq9@mail.sysu.edu.cn and E-Mail13322210199@163.com 
Wu et al.: P21 Mediates Resistance to SAHA-Induced Apoptosis by Inhibiting TRAIL Apoptotic Pathway

\section{Introduction}

AML (Acute Myeloid Leukemia, AML) is a highly heterogeneous disease. Only 20\% to $30 \%$ of AML patients enjoy long-term disease-free survival (DFS) after traditional anthracycline- and cytarabine-based induction regimens [1]. The majority of AML patients die because of refractory or relapsed disease [2,3]. To development novel agents for refractory or relapsed AML patient is urgently needed. SAHA(suberoylanilide hydroxamic acid), one of the HDACIs (Histone deacetylase inhibitors), has been shown in phase 1 clinical studies to be a promising agent that could be used in relapsed AML patients because of its favorable oral bioavailability and acceptable toxicity [4, 5]. However, SAHA showed different therapeutic effectiveness in treatment with AML patients. Recently a phase 2 clinical study of SAHA showed that a subset of AML patient was refractory to SAHA treatment [6]. Thus, knowledge of molecular basis for SAHA-mediated lethality is important for determining the most effective chemotherapeutic regimen and finding valuable biomarkers that can predict responsiveness in AML patients.

Induction of apoptosis is an important mechanism of chemotherapeutic drugs-induced cell death in transformed cells. SAHA is capable of inducing apoptotic cell death in leukemia cells by up-regulating the expression of certain genes. For example, SAHA promotes expression of TRAIL (TNF-related apoptosis-inducing ligand), a member of the TNF superfamily, by directly activating the TNFSF10 promoter. This triggers TRAIL-mediated apoptotic cell death in acute myeloid leukemia (AML) cells [7, 8]. Furthermore, a number of cell cycle regulators are affected by HDACI treatment. The cyclin-dependent kinase (CDK) inhibitor p21 is one of the most common cell cycle genes induced by SAHA $[9,10]$. SAHA is known to induce a specific increase in histone acetylation of H3K4 in the p21 promoter region and promote the expression of p21 mRNA and protein [9]. Whereas increased p21 expression is correlated with a block in proliferation, a number of reports have demonstrated that p21 can also affect apoptosis induced by HDACIs [11-13]. Andrew et al. reported that in human melanoma cells, p21 expression was up-regulated by histone deacetylase inhibitor ABHA (azelaicbis hydroxamic acid) and correlated with reduced sensitivity and a block of the apoptotic mechanism [12]. Cells that did not up-regulate p21 expression were sensitive to ABHA and died via apoptosis. In addition, Vrana et al. reported that p21-antisense cells were significantly more sensitive to SAHA-mediated apoptosis [11]. Although p21 seems to protect cells from SAHA-mediated apoptosis, the molecular mechanism by which p21 inhibits SAHA-mediated apoptosis is not well understood.

In the present study, we assessed the expression level of p21 and apoptotic rates after SAHA treatment in three human acute leukemia cell lines: HL60, NB4, and U937. Interestingly, we found that up-regulation of p21 blocked the TRAIL-mediated extrinsic apoptosis, which resulted in reduced sensitivity to SAHA. Conversely, p21-silencing by siRNA enhanced SAHAinduced apoptosis. Finally, we showed that the combined use of rapamycin down-regulated p21 and partially overcame p21-mediated resistance to SAHA. Our findings provided a novel mechanism that has critical implications for clinical AML treatment.

\section{Materials and Methods}

\section{Cell culture and viability assay}

The AML cell lines HL60, U937, and NB4 were purchased from the American Type Culture Collection and maintained in RPMI-1640 supplemented with 10\% fetal bovine serum (Hyclone), penicillin (100 units/mL; Sigma), and streptomycin (100 units/mL; Sigma). The cells were routinely maintained at $37^{\circ} \mathrm{C}$ in a humidified $5 \% \mathrm{CO}_{2}$ incubator. MTT assay was used to measure cell viability. Briefly, cells were plated in U-shape 96-well plates (JET BIOFIL) and then treated with the indicated treatments for $24 \mathrm{~h}$. To determine cell viability, $20 \mu \mathrm{l}$ of $10 \mathrm{mg} / \mathrm{ml}$ MTT (3-(4,4-dimethylthiazol-2-yl)-2,5-diphenyltetrazolium bromide; Sigma) was added to each well, and the plates were incubated for an additional $4 \mathrm{~h}$. After 1500 $\mathrm{rpm} \times 10 \mathrm{~min}$ centrifugation, the supernatant was removed, and the formed crystals were dissolved in $200 \mu \mathrm{l}$ 
Wu et al.: P21 Mediates Resistance to SAHA-Induced Apoptosis by Inhibiting TRAIL Apoptotic Pathway

of dimethyl sulphoxide ( igma). The absorbance $(\mathrm{A})$ was read at $495 \mathrm{~nm}$. Cell viability rate $(\%)=\mathrm{A}$ treated $/ \mathrm{A}$ control $\times 100 \%$.

\section{Plasmid constructs}

Theretrovirus construct MSCV-GFP was agift from professor Huanghua (NJhealth). Thep21 geneproduct was synthesized from the cDNA of NB4 cells using PrimeSTAR DNA polymerase (TAKARA) with the primers: 5'-ATCGTCAGATCTATGTCAGAACCGGCTGGG-3'(sense) and5'-AGGCTCGTCGACTTAGGGCTTCCTCTTGGAGA3'(antisense), and then subcloned into the MSCV-GFP vector.

\section{Viral infection}

Retroviruses containing MSCV-GFP and MSCV-P21 were packaged in GP2-293 cells by co-transfection with the envelope glycoprotein expression vector pVSV-G. Cells were infected with virus-containing media in the presence of $5 \mu \mathrm{g} / \mathrm{ml}$ polybrene (sigma) for $24 \mathrm{~h}$, and then positive cells were sorted by flow cytometry (Beckman).

\section{RNA interference}

Cells were cultured for 16 hours in 6-well plates before transfection. The siRNA oligonucleotides (sip21-1: 5'AGCGAUGGAACUUCGACUUTT-3', sip21-2:5'UGUCAGAACCGGCUGGGATT3', sip213: 5'GACC- AUGUGGACCUGUCACTT3', siTRAIL-1: 5'AACGAGCUGAAGCA- GAUGCAG3', siTRAIL-2: 5'GGCAUUCAUUCCUGAGCAATT3', siTRAIL-3: 5'GUCUAAAGAUGCAGAAUAUTT3', control:5'UUCUCCGAACGUGUCACGUTT3') were transfected into cells using Lipofectamine 2000 (Invitrogen) according to the manufacturer's instructions. To confirm down-regulation efficacy, targeted genes were detected by western blot.

\section{Apoptosis assay and cell cycle assay}

The cells were treated with indicated concentrations of SAHA for $24 \mathrm{~h}$. Apoptosis was measured using the AnnexinV-PE/7AAD apoptosis kits (KeyGen) according to the manufacturer's protocols. Data acquisition and analysis were performed using Cell Quest software via flowcytometry. For cell cycle analysis, cells were fixed in pre-cold ethanol, pelleted, resuspended in staining buffer $(0.2 \%$ TritonX-100, $0.1 \mathrm{mg} / \mathrm{ml}$ RNAse A, and $0.05 \mathrm{mg} / \mathrm{ml}$ propidium iodide), incubated at $-20^{\circ} \mathrm{C}$ overnight, pelleted, and resuspended in PBS before analysis. Cell cycle distribution was determined using the ModFit LT program (Verity Software).

\section{Western blot}

Western blot analysis was performed to detect p21, cleaved-PARP, TRAIL, caspase- 8 and $\beta$-actin expression. Antibodies were purchased from cell signaling technology. Briefly, cells were treated for $24 \mathrm{~h}$, then collected and washed with PBS. Cells were lysed with lysis buffer. $30 \mathrm{ug}$ of protein lysates was electrophoresed on SDS-PAGE gels and transferred to nitrocellulose membranes. The membranes were blocked, incubated with indicated primary antibodies at $4{ }^{\circ} \mathrm{C}$ overnight, and then conjugated with appropriate secondary antibodies. Protein bands were visualised with enhanced chemiluminescence.

\section{Statistical analysis}

Statistical analysis was performed using SPSS V. 17.0 (SPSS, Inc). The $\chi^{2}$ test and Student t-test were used to make statistical comparisons between groups. Data were presented as the mean \pm standard deviation (SD) and were representative of at least three independent experiments. Statistical tests were two-sided. P values $<0.05$ were considered to be statistically significant.

\section{Results}

Different acute leukemia cell lines show different sensitivity to SAHA

The AML cell lines HL60, U937 and NB4 were treated with the indicated concentrations of SAHA. Exposure to SAHA sharply diminished viable cell number of HL60 cells in a dose dependent manner, whereas SAHA exerted modest effect on NB4 and U937 cells (Fig. 1A). Consistently, cleavage of PARP was obviously detected throughout the concentration range 


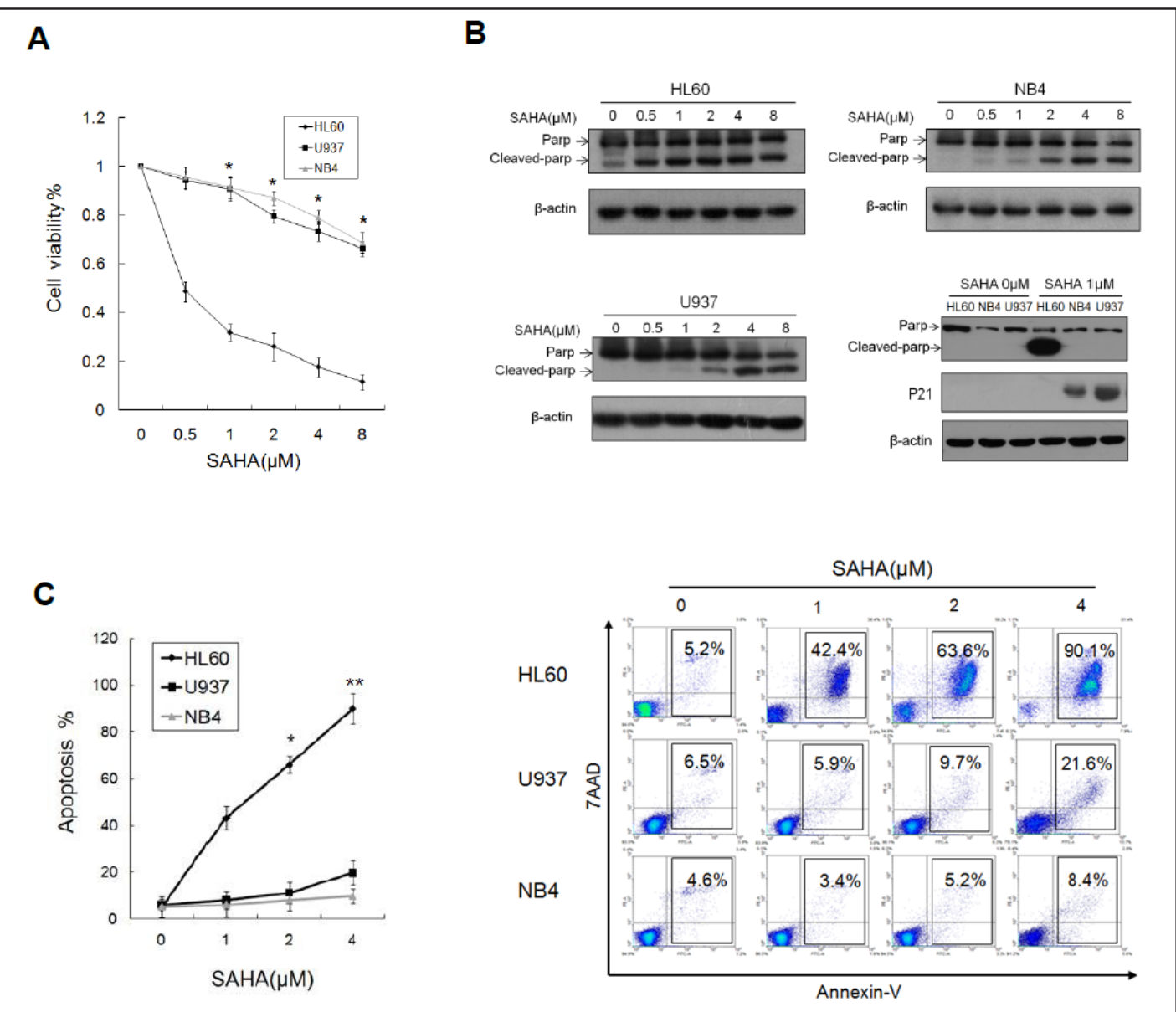

Fig. 1. Three acute leukemia cell lines were treated with SAHA. HL60, U937, and NB4 cells were treated with different doses of SAHA $(0,0.5,1,2,4,8 \mu \mathrm{M})$ for $24 \mathrm{~h}$. (A) Cell viability was assessed by MTT. Data are the mean \pm SD of three independent experiments. ${ }^{*} \mathrm{p}<0.05$. (B) Cell lysate was collected and assayed by western-blot with the indicated antibody. (C) Cell apoptosis rate was assessed by flow cytometry. Data are the mean \pm SD of three independent experiments. ${ }^{*} \mathrm{p}<0.05{ }^{* *} \mathrm{p}<0.01$.

Table 1. Three AML cell lines cell cycle profile. Three AML cell lines were exposed to indicated concentrations of SAHA and cell cycle distribution were analyzed by flow cytometry after $24 \mathrm{~h}$. Values were expressed as the mean percentage relative to the total cell population for three experiments \pm S.D.

tested $(0.5-8 \mu \mathrm{M})$ in HL60 cells, whereas in NB4 and U937 cells cleavage of PARP was delayed and detected at the dose of $\geq 2 \mu \mathrm{M}$ SAHA (Fig. 1B). To examine apoptosis induced by SAHA, apoptotic cell death was assessed by Annexin V/7AAD staining. As shown in Fig. 1C, after $24 \mathrm{~h}$ exposure to SAHA, the apoptotic population was sharply increased in HL60 cells in a dose dependent manner. In contrast, SAHA-mediated apoptotic rate was

\begin{tabular}{lccccc}
\hline Cell Type & Con. $(\mu M)$ & Sub-G1 & $G 1$ & $S$ & $G 2 / M$ \\
\hline HL60 & 0 & $3 \pm 2$ & $51 \pm 2$ & $37 \pm 1$ & $10 \pm 2$ \\
& 0.5 & $6 \pm 2$ & $45 \pm 1$ & $31 \pm 2$ & $18 \pm 1$ \\
& 1 & $39 \pm 2$ & $38 \pm 2$ & $11 \pm 1$ & $12 \pm 2$ \\
& 2 & $61 \pm 5$ & $22 \pm 2$ & $8 \pm 3$ & $9 \pm 1$ \\
U937 & 4 & $89 \pm 3$ & $10 \pm 3$ & $2 \pm 1$ & $2 \pm 1$ \\
& 8 & $95 \pm 1$ & $3 \pm 2$ & $1 \pm 1$ & $1 \pm 1$ \\
& 0 & $2 \pm 2$ & $50 \pm 3$ & $34 \pm 3$ & $12 \pm 2$ \\
& 0.5 & $6 \pm 1$ & $49 \pm 1$ & $30 \pm 2$ & $14 \pm 3$ \\
& 1 & $7 \pm 3$ & $47 \pm 1$ & $29 \pm 2$ & $17 \pm 2$ \\
NB4 & 2 & $10 \pm 2$ & $46 \pm 3$ & $24 \pm 3$ & $19 \pm 2$ \\
& 4 & $21 \pm 3$ & $45 \pm 2$ & $16 \pm 4$ & $20 \pm 2$ \\
& 8 & $24 \pm 3$ & $42 \pm 3$ & $12 \pm 2$ & $21 \pm 2$ \\
& 0 & $1 \pm 2$ & $50 \pm 2$ & $31 \pm 1$ & $18 \pm 2$ \\
& 0.5 & $5 \pm 1$ & $48 \pm 4$ & $30 \pm 2$ & $17 \pm 1$ \\
& 1 & $5 \pm 3$ & $49 \pm 3$ & $28 \pm 2$ & $18 \pm 2$ \\
& 2 & $7 \pm 3$ & $48 \pm 3$ & $25 \pm 3$ & $21 \pm 3$ \\
& 4 & $9 \pm 2$ & $46 \pm 2$ & $23 \pm 2$ & $22 \pm 2$ \\
& 8 & $11 \pm 3$ & $44 \pm 1$ & $21 \pm 2$ & $24 \pm 2$ \\
\hline
\end{tabular}


Fig. 2. The effect of $\mathrm{p} 21$ over-expression on SAHAinduced apoptosis. (A) The expression of p21 was confirmed by westernblot in HL60 cells over-expressing p21 (HL60P21) or its corresponding empty vector (HL60GFP) and $\beta$-actin was used as the loading control. (B) HL60P21 and HL60GFP cells were exposed to the indicated concentration of SAHA for $24 \mathrm{~h}$. Cell viability was analyzed by MTT assay. Columns, mean of triplicate determinations; bars, \pm SD. ${ }^{*} \mathrm{p}<0.05$. (C) Cell apoptosis rates were assessed by flow cytometry. Graphs showed mean values \pm S.D. for three independent experiments. ${ }^{*} \mathrm{p}<0.05$, ** $\mathrm{p}<0.01$. (D, E) After the treatment described in (B), 30 $\mu \mathrm{g}$ of protein was separated by SDS-PAGE and analyzed by western blot as described in Materials and Methods. Blots were probed with antibodies directed against p21, PARP, caspase-8, TRAIL, and $\beta$-actin to ensure equivalent loading and transfer.
A

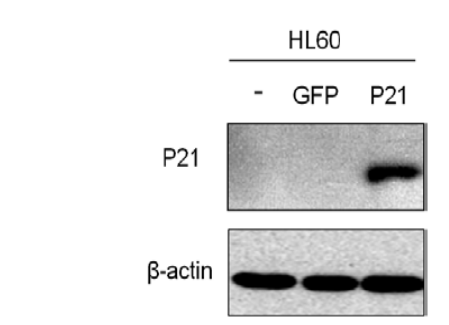

C

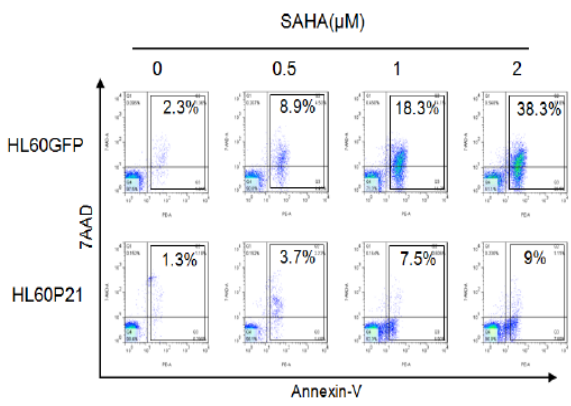

D

E

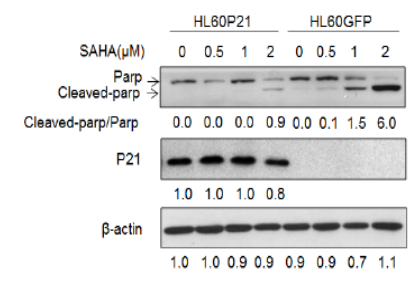

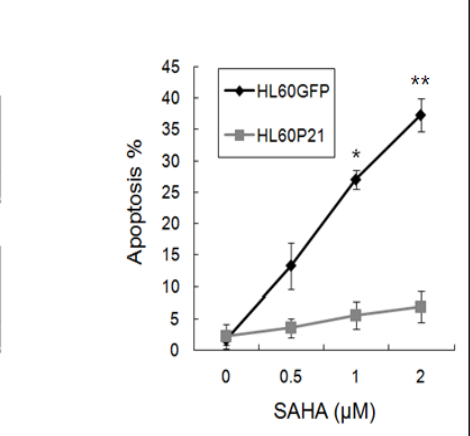

\section{B}
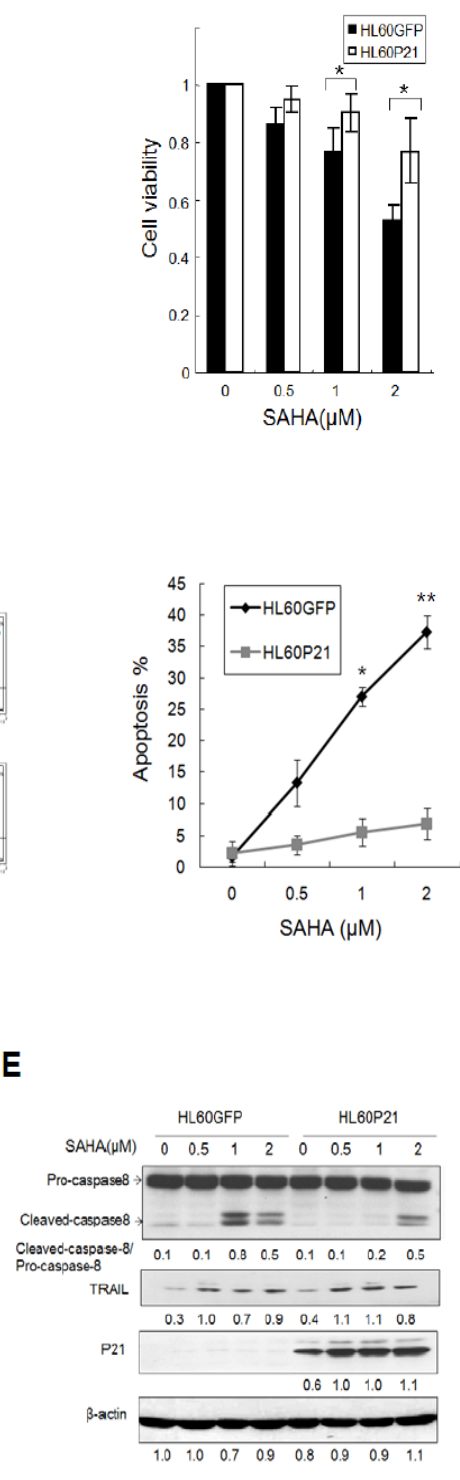

Table 2. HL60P 21 cell cycle profile. HL60 cells transfected with p21(HL60P21) and its corresponding controls (HL60GFP), were exposed to indicated concentrations of SAHA and cell cycle distribution were analyzed after $24 \mathrm{~h}$. Values were expressed as the mean percentage relative to the total cell population for three experiments \pm S.D.

\begin{tabular}{lccccc}
\hline Cell & Con. $(\mu M)$ & Sub-G1 & $G 1$ & $S$ & $G 2 / M$ \\
\hline HL60GFP & 0 & $2 \pm 1$ & $51 \pm 2$ & $36 \pm 1$ & $12 \pm 2$ \\
& 0.5 & $9 \pm 1$ & $47 \pm 3$ & $32 \pm 3$ & $11 \pm 3$ \\
& 1 & $21 \pm 3$ & $39 \pm 2$ & $24 \pm 2$ & $16 \pm 2$ \\
& 2 & $42 \pm 4$ & $30 \pm 2$ & $11 \pm 3$ & $17 \pm 3$ \\
HL60P21 & 0 & $1 \pm 1$ & $69 \pm 3$ & $27 \pm 2$ & $3 \pm 1$ \\
& 0.5 & $6 \pm 3$ & $67 \pm 4$ & $22 \pm 4$ & $4 \pm 1$ \\
& 1 & $11 \pm 3$ & $65 \pm 3$ & $18 \pm 2$ & $5 \pm 2$ \\
& 2 & $14 \pm 2$ & $66 \pm 2$ & $15 \pm 3$ & $4 \pm 1$ \\
\hline
\end{tabular}


A

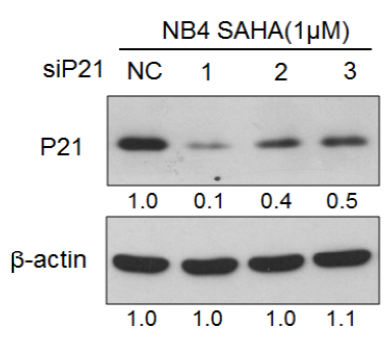

C

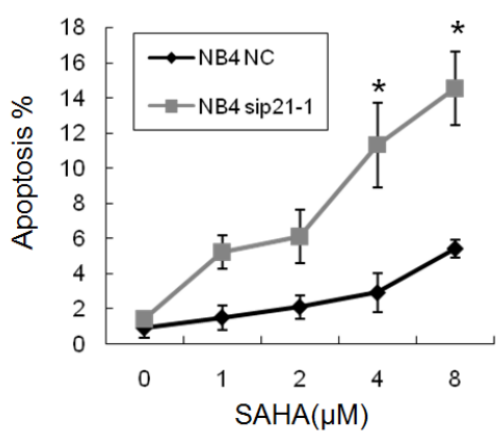

B

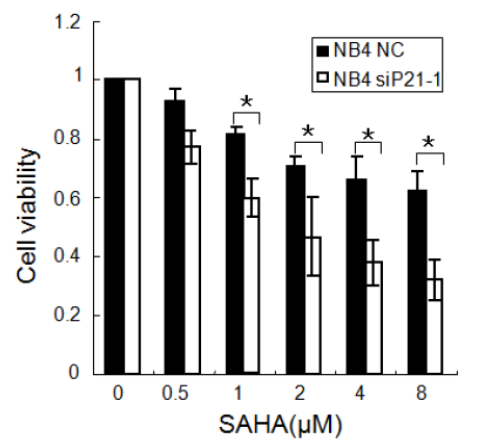

D

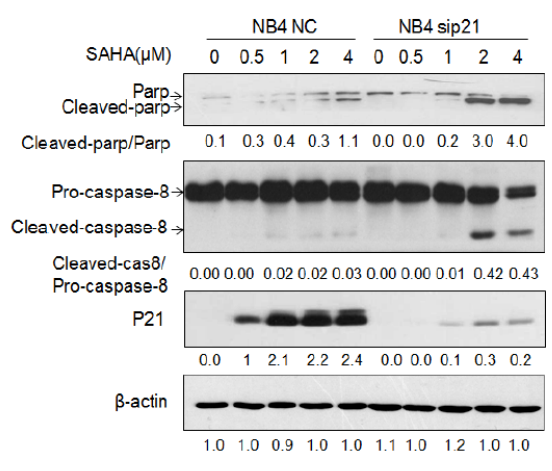

Fig. 3. The effect of p21-silencing on SAHA-induced apoptosis. (A) P21 was silenced by three target RNA sequences and one negative control sequence in NB4 cells. Western-blot was used to confirm silencing effects. (B) NB4 was treated by sequence 1 siRNA or negative control, and then, cells were exposed to the indicated concentrations of SAHA. MTT was used to assess cell viability. Data are the mean \pm SD of three independent experiments. ${ }^{*} p<0.05$. (C) Cell apoptosis rates were monitored by flow cytometry. Data are the mean $\pm S D$ of three independent experiments. ${ }^{*} \mathrm{p}<0.05$. (D) Cells were exposed to SAHA at the indicated concentrations for $24 \mathrm{~h}$, and then, cell lysate was analyzed by western blot with the indicated antibodies.

Table 3. NB4 sip21-24h cell cycle profile. NB4 cells transfected with p21 siRNA and negative control siRNA, were exposed to indicated concentrations of SAHA and cell cycle distribution analyses after $24 \mathrm{~h}$. Values were expressed as the mean percentage relative to the total cell population for three experiments \pm S.D.

\begin{tabular}{lccccc}
\hline Cell & Con. $(\mu M)$ & Sub-G1 & $G 1$ & $S$ & $G 2 / M$ \\
\hline NB4 NC & 0 & $2 \pm 1$ & $50 \pm 4$ & $32 \pm 1$ & $18 \pm 2$ \\
& 1 & $2 \pm 1$ & $48 \pm 3$ & $31 \pm 2$ & $19 \pm 2$ \\
& 2 & $3 \pm 2$ & $47 \pm 3$ & $31 \pm 2$ & $21 \pm 3$ \\
& 4 & $4 \pm 2$ & $45 \pm 5$ & $30 \pm 3$ & $22 \pm 2$ \\
& 8 & $5 \pm 3$ & $43 \pm 4$ & $29 \pm 3$ & $24 \pm 4$ \\
NB4 sip21 & 0 & $1 \pm 1$ & $49 \pm 4$ & $35 \pm 2$ & $16 \pm 2$ \\
& 1 & $6 \pm 3$ & $43 \pm 3$ & $34 \pm 2$ & $19 \pm 3$ \\
& 2 & $7 \pm 3$ & $41 \pm 3$ & $33 \pm 3$ & $20 \pm 3$ \\
& 4 & $12 \pm 4$ & $33 \pm 3$ & $29 \pm 3$ & $24 \pm 4$ \\
& 8 & $19 \pm 3$ & $29 \pm 3$ & $24 \pm 2$ & $27 \pm 3$ \\
\hline
\end{tabular}

fractions in a dose dependent manner, whereas the subdiploid population displayed a modest expansion in U937 and NB4 cells at the same concentration (Table 1). The NB4 and U937 cells experienced a decrease in the G0/G1 and S phase population butan increase in the G2/M population, indicating that SAHA induced apoptosis in the G0/G1 and S phase cell populations in these cells. Interestingly, SAHA treatment induced obvious p21expression in U937 and NB4 cells but not in HL60cells (Fig. 1B). These results suggested that U937 and NB4 cells were more resistant to SAHA-mediated apoptosis and p21 expression might be an important factor that led to SAHA resistance in these cells. 
Wu et al.: P21 Mediates Resistance to SAHA-Induced Apoptosis by Inhibiting TRAIL Apoptotic Pathway

Fig. 4. The effect of p21 over-expression on TRAILinduced apoptosis. (A) HL60GFP and HL60P21 cells were treated with recombinant TRAIL at the concentration of $0,2,4,8$, 16 , or $32 \mathrm{ng} / \mathrm{ml}$ for $24 \mathrm{~h}$. Cell viability was measured by MTT. Graphs show mean values \pm S.D. of three independent experiments. ${ }^{*} \mathrm{p}<0.05$. (B) Following the above treatment, the percentages of apoptotic cells were measured by flow cytometry. Columns, mean of triplicate determinations; bars, \pm SD. * $\mathrm{p}<0.05$, ** $\mathrm{p}<0.01$. (C) Following the above treatment, Western blot was used to detect cleaved-PARP, procaspase-8, cleaved-caspase-8 and p21.
A

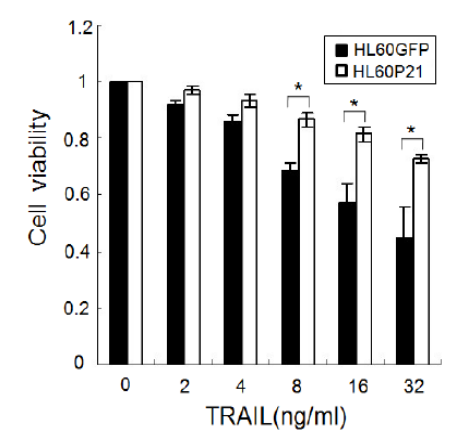

B

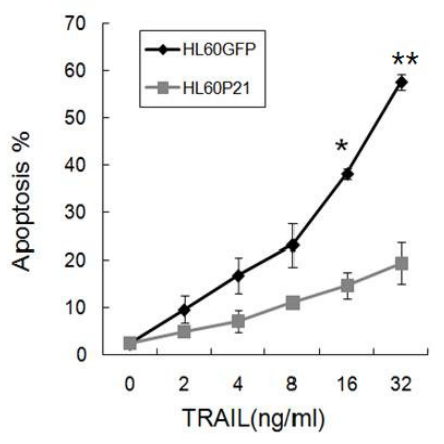

C

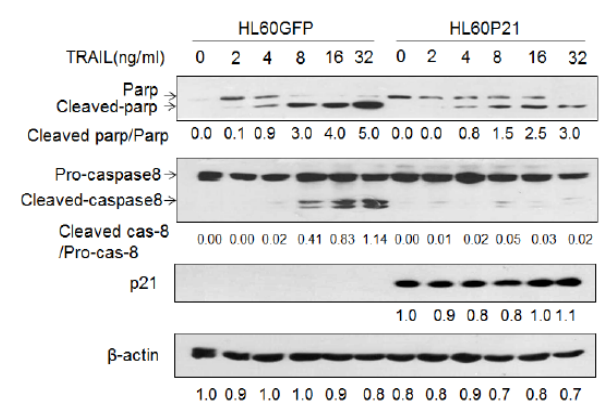

Over-expression of p21 protects HL60 cells from SAHA-induced apoptosis and caspase-8 cleavage

To assess the functional role of p21 in SAHA-related apoptosis, we established p21 over-expression HL60 cells (HL60P21) and appropriate control cells (HL60GFP) using the retrovirus plasmid MSCV-GFP. Fig. 2A showed that the p21 was clearly over-expressed in HL60P21 cells compared to HL60GFP cells. Cells were treated with SAHA the indicated concentrations for $24 \mathrm{~h}$. Over-expression of $\mathrm{p} 21$ significantly prevented SAHA-mediated loss of cell viability by $16.7 \%(1 \mu \mathrm{M})$ and $23.9 \%(2 \mu \mathrm{M}, \mathrm{P}<0.05)$ respectively (Fig. $2 \mathrm{~B})$. A consistent result was also observed in the apoptosis assay. The apoptotic rate was reduced nearly three-fold $(1 \mu \mathrm{M})$ and five-fold $(2 \mu \mathrm{M})$ in HL60P21 cells compared to control cells respectively (Fig. 2C). Furthermore, cleaved caspase-8 and PARP were clearly detected in control cells (HL60GFP) but not in HL60P21 cells at the dose of $1 \mu \mathrm{M}$. However they were detected in both cells at the dose of $2 \mu \mathrm{M}$. This indicated that p21 delayed caspase- 8 and PARP cleavage upon SAHA treatment (Fig. 2D and E). Previous reports had shown TRAIL was involved in SAHA mediated apoptosis. We found that following exposure of cells to SAHA, TRAIL expression was increased over basal levels both in HL60GFP and HL60P21 cells. Nevertheless, p21 over-expression exerted little effect on TRAIL expression level. FACS analysis showed that HL60P21 cells were arrested in the G1 phase, as demonstrated by the reduction in S and G2/M phase cells. Enforced expression of p21 afforded significant protection against SAHAinduced apoptosis which was normally indicated by the presence of cells with $<2 \mathrm{n}$ DNA content (Table 2). In conclusion, these data suggested that $\mathrm{p} 21$ could protect AML cells from SAHA-mediate apoptosis and caspase- 8 cleavage, most probably by G1 phase arrest.

Down-regulation of 21 enhances SAHA-induced apoptosis and caspase-8 cleavage in NB4

To evaluate whether silencing p21 could sensitize NB4 cells to SAHA, a NB4 cell line transfected with double strand p21-antisense siRNA was employed. As anticipated, p21 levels were dramatically decreased in p21 antisense-expressing cells compared to negative 
Fig. 5. The effect of TRAIL silencing on SAHA-mediated apoptosis. (A) Double strand RNA specifically targeting TRAIL and control RNA were transfected into HL60 cells (HL60 siT and HL60 NC). Cells were treated with the indicated concentrations of SAHA. After treatment, cells were harvested for MTT assay. Columns, mean of triplicate determinations; bars, \pm SD. *
A

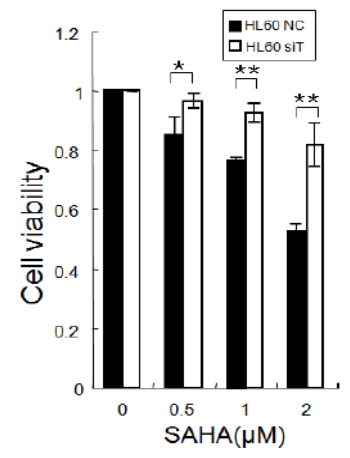

B

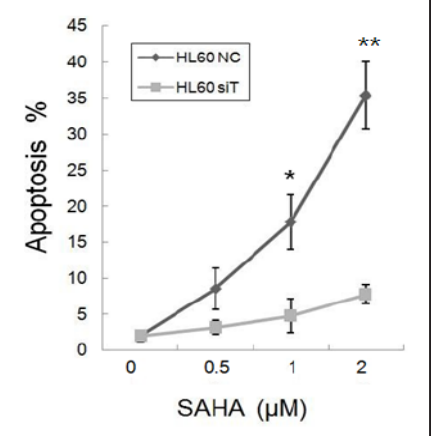

C

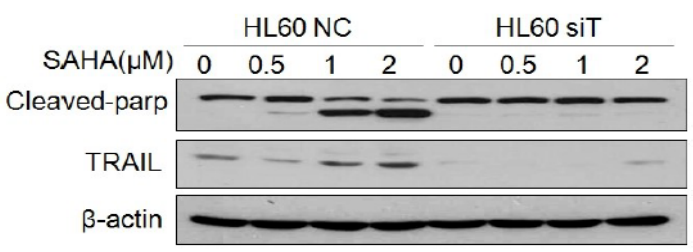

$\mathrm{p}<0.05,{ }^{* *} \mathrm{p}<0.01$ (B) After the above treatment, the percentage of AnnexinV-stained apoptotic cells was measured by flow cytometry. Graphs showed mean values \pm S.D. of three independent experiments. ${ }^{*} \mathrm{p}<$ 0.05 , ${ }^{* *} \mathrm{p}<0.01$. (C) After the above treatment, cells were collected for immunoblot analysis for PARP and TRAIL and $\beta$-actin was used as a loading control.

Table 4. HL60-siTRAIL cell cycle profile. HL60 cells transfected with TRAIL siRNA and negative control SiRNA, were exposed to indicated concentrations of SAHA and cell cycle distribution analyses after $24 \mathrm{~h}$. Values were expressed as the mean percentage relative to the total cell population for three experiments \pm S.D.

\begin{tabular}{lccccc}
\hline Cell & Con. $(\mu M)$ & Sub-G1 & $G 1$ & $S$ & G2/M \\
\hline HL60 NC & 0 & $2 \pm 1$ & $52 \pm 3$ & $37 \pm 3$ & $11 \pm 1$ \\
& 0.5 & $11 \pm 2$ & $47 \pm 2$ & $31 \pm 2$ & $12 \pm 2$ \\
& 1 & $17 \pm 3$ & $43 \pm 3$ & $22 \pm 2$ & $16 \pm 2$ \\
& 2 & $36 \pm 5$ & $39 \pm 3$ & $12 \pm 2$ & $17 \pm 4$ \\
HL60 siT & 0 & $1 \pm 1$ & $50 \pm 3$ & $38 \pm 2$ & $12 \pm 3$ \\
& 0.5 & $4 \pm 2$ & $48 \pm 5$ & $37 \pm 3$ & $11 \pm 2$ \\
& 1 & $5 \pm 2$ & $46 \pm 2$ & $32 \pm 4$ & $16 \pm 3$ \\
& 2 & $7 \pm 3$ & $45 \pm 2$ & $30 \pm 3$ & $15 \pm 3$ \\
\hline
\end{tabular}

control cells upon SAHA(1 $\mu \mathrm{M})$ treatment (Fig. 3A). P21-antisense cells were significantly more sensitive to SAHA-mediated toxicity at increasing SAHA concentrations, leading to $23.5 \%(1 \mu \mathrm{M}), 28.9 \%(2 \mu \mathrm{M}), 31.5 \%(4 \mu \mathrm{M})$ and $32.9 \%(8 \mu \mathrm{M}, \mathrm{p}<0.05)$ reduction in cell viability respectively (Fig. 3B). The apoptosis induced by SAHA was significantly increased in the p21-antisense cells at $\geq 4 \mu \mathrm{M}$ SAHA concentrations ( $p<0.05$, Fig. $3 \mathrm{C})$. Consistent with those findings, the extent of caspase- 8 and PARP cleavage was obviously enhanced at $\geq 2 \mu \mathrm{M}$ SAHA concentrations in p21-antisense cells (Fig. 3D). Furthermore, p21-antisense cells significantly enhanced SAHA-mediated expansion in the sub-G1 phase populations at $\geq 4 \mu \mathrm{M}$ SAHA concentrations in FACS assay (Table 3). From these results, we concluded that p21 knockdown enhanced SAHA-induced apoptosis and caspase-8 cleavage in NB4 cells. 
Wu et al.: P21 Mediates Resistance to SAHA-Induced Apoptosis by Inhibiting TRAIL Apoptotic Pathway

Fig. 6. Combined treatment of SAHA and rapamycin in NB4 and U937 cells. (A),(B) NB4 and U937 cells were exposed to SAHA $(1 \mu \mathrm{mol} / \mathrm{L})$ and rapamycin (100nmol/L) either alone or in combination for $24 \mathrm{~h}$, after which cells were harvested for MTT. Columns, mean of triplicate determinations; bars, \pm SD. ${ }^{*} \mathrm{p}$ $<0.05$. (C) After the above treatment, apoptotic cells were monitored by flow cytometry. Columns, mean of triplicate determinations; bars, \pm SD. * $\mathrm{p}<0.05$. (D),(E) After the treatment described in (A) and (B), western blot was used to detect cleaved-PARP, TRAIL, caspase-8, p21 and $\beta$-actin to ensure equivalent loading and transfer.

\section{A}
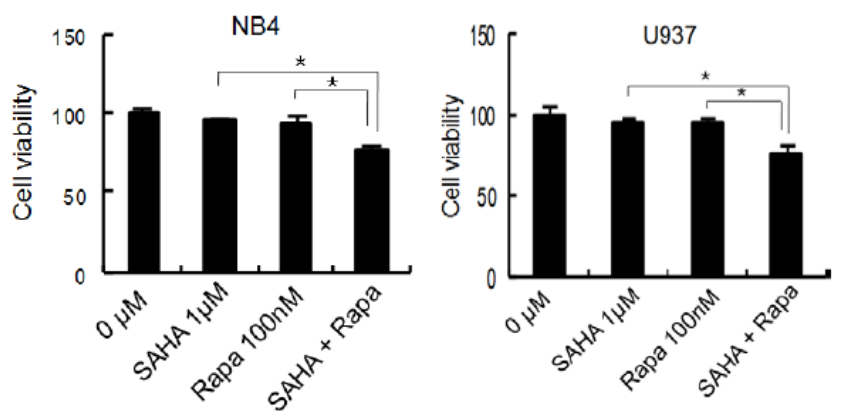

B
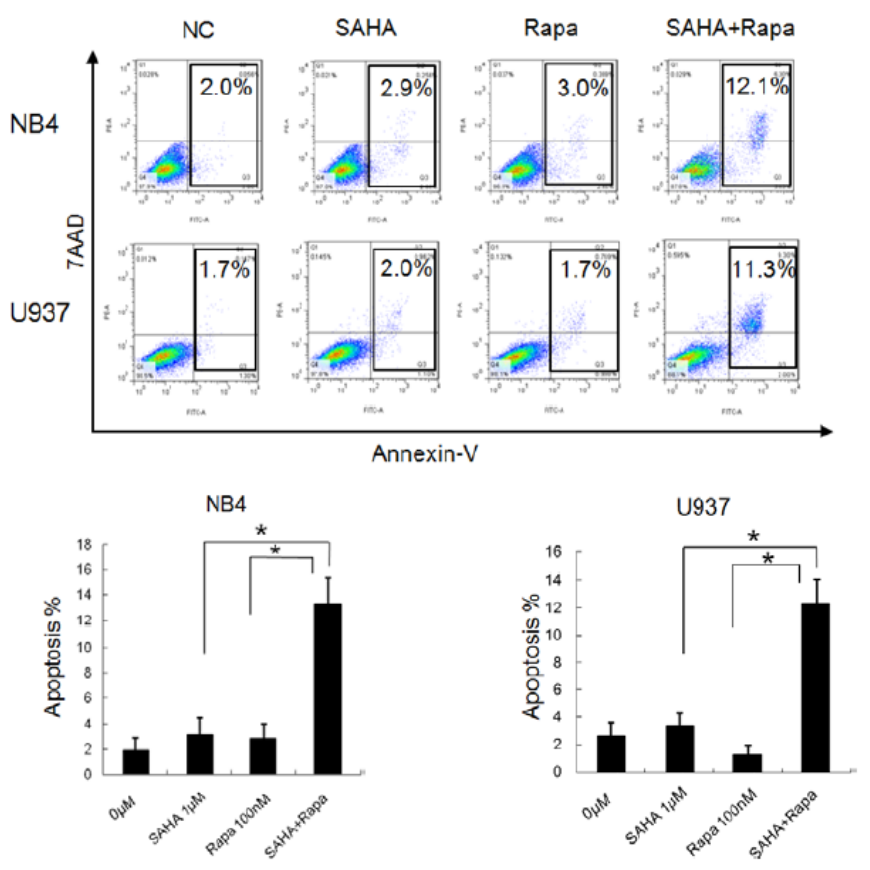

C

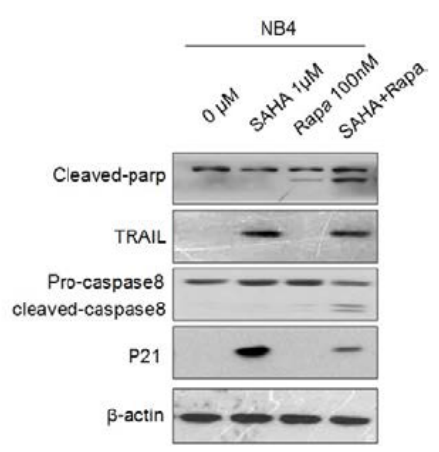

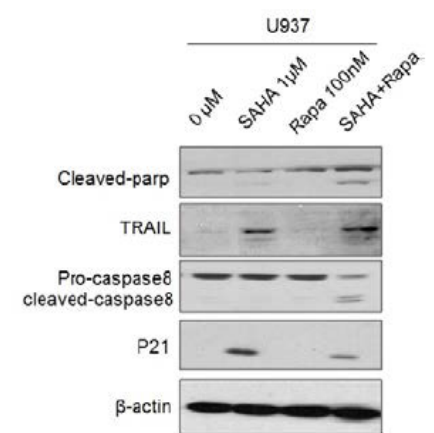

Overexpression of P21 inhibits TRAIL-induced caspase-8 cleavage and apoptosis

It is well-known that caspase-8 is an important downstream caspase in the TRAILmediated apoptotic pathway. To determine whether p21 inhibit the apoptosis of AML cell through TRAIL-mediated apoptotic pathway, p21 over-expression cells (HL60P21) and control cells (HL60GFP) were treated with indicated concentrations of recombinant TRAIL protein for $24 \mathrm{~h}$. Enforced expression of p21 significantly protected cells from lose of cell viability by $20.5 \%(8 \mathrm{ng} / \mathrm{ml}), 24.8 \%(16 \mathrm{ng} / \mathrm{ml})$ and $32.5 \%(32 \mathrm{ng} / \mathrm{ml})$ respectively (Fig. 
Wu et al.: P21 Mediates Resistance to SAHA-Induced Apoptosis by Inhibiting TRAIL Apoptotic Pathway

4A). Consistently, the apoptosis of p21 over-expression cells was reduced by $25.7 \%$ (16 ng/ $\mathrm{ml})$ and $44.3 \%(32 \mathrm{ng} / \mathrm{ml})$ respectively. Finally, the cleavage of PARP and caspase-8 were notably inhibited in p21 over-expressing cells at $\geq 8 \mathrm{ng} / \mathrm{ml}$ TRAIL concentrations (Fig. 4C). In conclusion, these results suggested that TRAIL-mediated apoptosis was inhibited by overexpression of p21 in AML cells.

TRAIL silencing results in reduced sensitivity to SAHA

Previous studies suggested that the TRAIL induction accounted for the cancer selective apoptotic activity of HDACIs. HDACI MS275-induced apoptosis resulted from activation of the extrinsic TRAIL-dependent pathway [7]. To assess specific contributions of TRAIL to SAHA-induced growth-regulatory effects in AML cells, we knocked down the expression of TRAIL by RNA interference. As shown in Fig. 5A and 5B, silencing of TRAIL significantly inhibited the lethal and apoptotic activity of SAHA at $\geq 1 \mu \mathrm{M}$ SAHA concentrations. Consistently, silencing of TRAIL blocked PARP cleavage (Fig. 5C) and reduced SAHA-mediated sub-G1 expansion throughout the concentration range tested $(0.5-2 \mu \mathrm{M})$ (Table 4).Together these results identified TRAIL as possible key mediators of SAHA-induced apoptosis in AML cells.

Rapamycin down-regulates $p 21$ and sensitizes NB4 and U937 to SAHA

Recent studies in the literature reported that mTOR inhibitor rapamycin enhanced the effect of luteolin with a concomitant p21 inhibition [14]. Based on the preceding results, we predicted that rapamycin might sensitize NB4 and U937 cells to SAHA-mediated cell death by inhibition of p21. To test this hypothesis, we evaluated the potential synergistic effect of SAHA in combination with rapamycin in NB4 and U937 cells. As shown in Fig. 6A, the combination treatment of SAHA plus rapamycin induced a statistically significant decrease in the cell viability compared to SAHA or rapamycin alone. Consistently, the apoptotic assay showed that combined treatment induced an increased apoptotic rate in NB4 and U937 cells by $9.1 \%$ and $9.6 \%$ respectively (Fig. 6B). In addition, p21 level was down-regulated with a concomitant of enhanced caspase-8 and PARP cleavage after combined treatment (Fig. 6C). These results suggested that combination of rapamycin might be a promising strategy to increase the efficacy of SAHA-based treatment against AML cells.

\section{Discussion}

Herein we reported that different AML cell lines displayed different sensitivity to SAHA treatment. After $24 \mathrm{~h}$ of SAHA treatment, HL60 cells were more sensitive to SAHA-induced apoptosis than U937 and NB4 cells. Interestingly, we detected high expression level of p21 in U937 and NB4 cells but not in HL60 cells. Accumulating evidence has shown that p21 is a modulator of apoptotic pathways that can induce either pro-apoptotic or anti-apoptotic responses [15-21]. The induction of a particular response in transformed cells seems to depend on "cell content", such as molecular changes, cell and HDACI types. HDACI apicidin upregulated the expression of p21 in HL60 cells and contributed to the induction of apoptosis [22]. In contrast, up-regulated p21 expression reduced sensitivity to ABHA by blockage of apoptosis in human melanoma cells [12]. Consistently, antisense ablation of p21 enhanced SAHA-related lethality [11]. To confirm the anti-apoptotic effect of p21 in response to SAHA, we transfected HL60 cells with recombinant p21. As expected, Enforced expression of p21 in HL60 cells afforded significant protection against SAHA-induced apoptosis and toxicity. P21 over-expression cells were arrested in the G1 phase, concomitant with a decrease in S phase content (Table 2). Given the notion that $\mathrm{S}$ phase cells are more vulnerable to SAHA-mediated cell death, the G1 phase arrest may contribute to the anti-apoptotic effect of p21. Conversely, Antisense ablation of p21 expression in NB4 cells significantly enhanced SAHA-induced apoptosis and lethality. These results indicated that in AML cells p21 was an important factor that blocked the apoptotic mechanism induced by SAHA. 
Wu et al.: P21 Mediates Resistance to SAHA-Induced Apoptosis by Inhibiting TRAIL Apoptotic Pathway

A number of mechanisms may contribute to the anti-apoptotic effect of p21, including inhibition of caspase-3, caspase-9 and cyclin A/Cdk2 complex, a requisite effector of apoptotic death [16, 17, 23, 24]. P21 directly bound and inactivated procaspase-3, resulting in resistance to Fas-mediated apoptosis [16]. IR (ionizing radiation) induced caspase-9 activation was abrogated by p21 in colon cancer cells [17]. We observed that after enforced expression of p21 in HL60 cells, the cleavage of caspase-8 and PARP were delayed, whereas silencing of p21 in NB4 cells resulted in obviously enhanced caspase-8 and PARP cleavage. Caspase-8 is responsible for the proteolytic cleavage events associated with extrinsic apoptosis, including PARP cleavage. The delayed cleavage of PARP was most likely to be a consequence of the inhibition of caspase- 8 activation. These results suggested that reduced ability to rapidly cleave caspase-8 and PARP was closely correlated with up-regulated p21 expression in those cells, indicating that p21 exerted anti-apoptotic effect in AML cell lines via blockage of caspase-8 dependent extrinsic pathway.

Caspase-8 is an important downstream effector of the TRAIL apoptotic pathway [25]. TRAIL is a member of the TNF family of apoptosis-inducing proteins that initiates apoptosis in a variety of cancer cells [26-28]. TRAIL-receptor ligation recruits and activates pro-caspase-8, which leads to initiation of the extrinsic apoptotic pathway. The extrinsic apoptotic pathway accounts for HDACI-induced cell death in many transformed cells [8, 29, 30]. Previous studies revealed that SAHA induced expression of TRAIL by directly activating its promoter and triggering TRAIL-mediated apoptosis in acute myeloid leukemia cells [29]. This was consistent with our finding that TRAIL expression was up-regulated upon SAHA treatment (Fig. 2E). Antisense ablation of TRAIL in the sensitive HL60 cells significantly reduced SAHA-mediated apoptotic and cytotoxic effects, indicating that TRAIL signaling pathway was important for SAHA pharmacological action.

Previous reports showed that TRAIL-induced apoptosis was associated with p21 levels $[31,32]$. But the role of p21 in regulating TRAIL-mediated apoptosis was controversial. P21 was required for HDACI sodium butyrate and TRAIL-induced apoptosis in breast cancer cells [31]. In contrast, over-expression of p21, or its N-terminal 91 amino acids containing cell cycle-inhibitory activity inhibited TRAIL receptor DR4-dependent caspase cleavage in colon cancer cell lines [32]. We examined whether TRAIL-induced apoptosis was regulated by p21 in AML cells. The results showed that TRAIL-mediated apoptosis and lethality were significantly reduced by p21 over-expression (Fig. 4A and 4B). Notably the cleavage of caspase- 8 and PARP was blocked throughout the TRAIL concentration range in p21 over-expression cells (Fig. 4C). The reduced cleavage of PARP was most likely to be a consequence of the inhibition of caspase- 8 activation by p21. Nevertheless, certain extent of PARP cleavage was detected in control cells for the probable reason that other upstream caspases participated in the TRAIL apoptotic pathway. Moreover, p21 over-expression exerted minimal effect on TRAIL expression level in HL60 cells. The data presented here suggested that p21 up-expression blocked caspase-8 cleavage to suppress TRAIL-mediated apoptosis, which might be an important reason that reduced SAHA-mediated apoptosis in AML cells. These results indicate that use of SAHA as a chemotherapeutic drug may be more effective in AML treatment when it does not induce p21 expression.

Recent studies reported that mTOR inhibitor rapamycin enhanced the effect of luteolin with a concomitant p21 inhibition [14]. Rapamycin is known to inhibit the mammalian target of rapamycin (mTOR) protein kinase, but its role in controlling tumour sensitivity is poorly understood [33]. We found that co-treatment of SAHA with rapamycin downregulated p21 and increased the sensitivity of AML cells to SAHA, which was accompanied by cleavage of PARP and caspase-8. These findings provide evidence that rapamycin may partially overcome p21-mediated resistance to SAHA in AML treatment.

In summary, we reported here that p21 functioned as an anti-apoptotic modulator through suppression of the TRAIL apoptotic pathway, which led to resistance to SAHA in AML cell lines. Moreover, we found that combined treatment with SAHA and rapamycin might be effective in clinical AML treatment. Our results give a new insight into the mechanism by which p21 suppresses apoptosis and enhances cell survival in SAHA-treated AML cells. 
Wu et al.: P21 Mediates Resistance to SAHA-Induced Apoptosis by Inhibiting TRAIL Apoptotic Pathway

These findings provide evidences for $\mathrm{p} 21$ as a candidate biomarker to predict response and a potential target for SAHA-based therapy in the treatment of AML patients.

\section{Acknowledgements}

We thank other members of the Quentin Liu lab for their critical comments and technical support. We thank Prof. HuangHua (NJ health) for kindly providing the MSCV-GFP plasmids. This research work was supported by the National Basic Research Program of China (973 Program; No. 2012CB967000 and 2011CB504300 to Q.L.) and, National Natural Science Foundation of China (81130040 to Q.L.).

\section{Disclosure Statement}

The authors declare no competing financial interest.

\section{References}

1 Kumar CC: Genetic abnormalities and challenges in the treatment of acute myeloid leukemia. Genes Cancer 2011;2:95-107.

2 Farag SS, Ruppert AS, Mrozek K, Mayer RJ, Stone RM, Carroll AJ, Powell BL, Moore JO, Pettenati MJ, Koduru PR, Stamberg J, Baer MR, Block AW, Vardiman JW, Kolitz JE, Schiffer CA, Larson RA, Bloomfield CD:

Outcome of induction and postremission therapy in younger adults with acute myeloid leukemia with normal karyotype: A cancer and leukemia group b study. J Clin Oncol 2005;23:482-493. Klepin HD, Balducci L: Acute myelogenous leukemia in older adults. Oncologist 2009;14:222-232. Garcia-Manero G, Yang H, Bueso-Ramos C, Ferrajoli A, Cortes J, Wierda WG, Faderl S, Koller C, Morris G, Rosner G, Loboda A, Fantin VR, Randolph SS, Hardwick JS, Reilly JF, Chen C, Ricker JL, Secrist JP, Richon VM, Frankel SR, Kantarjian HM: Phase 1 study of the histone deacetylase inhibitor vorinostat (suberoylanilide hydroxamic acid [saha]) in patients with advanced leukemias and myelodysplastic syndromes. Blood 2008;111:1060-1066.

5 Gojo I, Tan M, Fang HB, Sadowska M, Lapidus R, Baer MR, Carrier F, Beumer JH, Anyang BN, Srivastava RK, Espinoza-Delgado I, Ross DD: Translational phase i trial of vorinostat (suberoylanilide hydroxamic acid) combined with cytarabine and etoposide in patients with relapsed, refractory, or high-risk acute myeloid leukemia. Clin Cancer Res 2013;19:1838-1851.

-6 Schaefer EW, Loaiza-Bonilla A, Juckett M, DiPersio JF, Roy V, Slack J, Wu W, Laumann K, Espinoza-Delgado I, Gore SD: A phase 2 study of vorinostat in acute myeloid leukemia. Haematologica 2009;94:1375-1382.

7 Nebbioso A, Clarke N, Voltz E, Germain E, Ambrosino C, Bontempo P, Alvarez R, Schiavone EM, Ferrara F, Bresciani F, Weisz A, de Lera AR, Gronemeyer H, Altucci L: Tumor-selective action of hdac inhibitors involves trail induction in acute myeloid leukemia cells. Nat Med 2005;11:77-84.

-8 Nakata S, Yoshida T, Horinaka M, Shiraishi T, Wakada M, Sakai T: Histone deacetylase inhibitors upregulate death receptor 5/trail-r2 and sensitize apoptosis induced by trail/apo2-l in human malignant tumor cells. Oncogene 2004;23:6261-6271.

-9 Richon VM, Sandhoff TW, Rifkind RA, Marks PA: Histone deacetylase inhibitor selectively induces p21waf1 expression and gene-associated histone acetylation. Proc Natl Acad Sci U S A 2000;97:10014-10019.

10 Peart MJ, Smyth GK, van Laar RK, Bowtell DD, Richon VM, Marks PA, Holloway AJ, Johnstone RW: Identification and functional significance of genes regulated by structurally different histone deacetylase inhibitors. Proc Natl Acad Sci U S A 2005;102:3697-3702.

11 Vrana JA, Decker RH, Johnson CR, Wang Z, Jarvis WD, Richon VM, Ehinger M, Fisher PB, Grant S: Induction of apoptosis in u937 human leukemia cells by suberoylanilide hydroxamic acid (saha) proceeds through pathways that are regulated by bcl-2/bcl-xl, c-jun, and p21cip1, but independent of p53. Oncogene 1999;18:7016-7025.

12 Burgess AJ, Pavey S, Warrener R, Hunter LJ, Piva TJ, Musgrove EA, Saunders N, Parsons PG, Gabrielli BG: Up-regulation of p21(waf1/cip1) by histone deacetylase inhibitors reduces their cytotoxicity. Mol Pharmacol 2001;60:828-837. 
Wu et al.: P21 Mediates Resistance to SAHA-Induced Apoptosis by Inhibiting TRAIL Apoptotic Pathway

13 Saito A, Yamashita T, Mariko Y, Nosaka Y, Tsuchiya K, Ando T, Suzuki T, Tsuruo T, Nakanishi O: A synthetic inhibitor of histone deacetylase, ms-27-275, with marked in vivo antitumor activity against human tumors. Proc Natl Acad Sci U S A 1999;96:4592-4597.

14 Chiang CT, Way TD, Lin JK: Sensitizing her2-overexpressing cancer cells to luteolin-induced apoptosis through suppressing p21(waf1/cip1) expression with rapamycin. Mol Cancer Ther 2007;6:2127-2138.

15 Asada M, Yamada T, Ichijo H, Delia D, Miyazono K, Fukumuro K, Mizutani S: Apoptosis inhibitory activity of cytoplasmic p21(cip1/waf1) in monocytic differentiation. EMBO J 1999;18:1223-1234.

16 Suzuki A, Tsutomi Y, Akahane K, Araki T, Miura M: Resistance to fas-mediated apoptosis: Activation of caspase 3 is regulated by cell cycle regulator p21waf1 and iap gene family ilp. Oncogene 1998;17:931-939.

17 Sohn D, Essmann F, Schulze-Osthoff K, Janicke RU: P21 blocks irradiation-induced apoptosis downstream of mitochondria by inhibition of cyclin-dependent kinase-mediated caspase- 9 activation. Cancer Res 2006;66:11254-11262.

18 Aneja R, Ghaleb AM, Zhou J, Yang VW, Joshi HC: P53 and p21 determine the sensitivity of noscapineinduced apoptosis in colon cancer cells. Cancer Res 2007;67:3862-3870.

19 Hassani S, Ghaffari SH, Zaker F, Mirzaee R, Mardani H, Bashash D, Zekri A, Yousefi M, Zaghal A, Alimoghaddam K, Ghavamzadeh A: Azidothymidine hinders arsenic trioxide-induced apoptosis in acute promyelocytic leukemia cells by induction of $\mathrm{p} 21$ and attenuation of g2/m arrest. Ann Hematol 2013;92:1207-1220.

20 Suzuki H, Yabuta N, Okada N, Torigata K, Aylon Y, Oren M, Nojima H: Lats2 phosphorylates p21/cdkn1a after uv irradiation and regulates apoptosis. J Cell Sci 2013;126:4358-4368.

21 Gartel AL, Tyner AL: The role of the cyclin-dependent kinase inhibitor p21 in apoptosis. Mol Cancer Ther 2002;1:639-649.

22 Kwon SH, Ahn SH, Kim YK, Bae GU, Yoon JW, Hong S, Lee HY, Lee YW, Lee HW, Han JW: Apicidin, a histone deacetylase inhibitor, induces apoptosis and fas/fas ligand expression in human acute promyelocytic leukemia cells. J Biol Chem 2002;277:2073-2080.

23 Adachi S, Ito H, Tamamori-Adachi M, Ono Y, Nozato T, Abe S, Ikeda M, Marumo F, Hiroe M: Cyclin a/cdk2 activation is involved in hypoxia-induced apoptosis in cardiomyocytes. Circ Res 2001;88:408-414.

24 Jin YH, Yoo KJ, Lee YH, Lee SK: Caspase 3-mediated cleavage of p21waf1/cip1 associated with the cyclin a-cyclin-dependent kinase 2 complex is a prerequisite for apoptosis in sk-hep-1 cells. J Biol Chem 2000;275:30256-30263.

25 Seol DW, Li J, Seol MH, Park SY, Talanian RV, Billiar TR: Signaling events triggered by tumor necrosis factorrelated apoptosis-inducing ligand (trail): Caspase- 8 is required for trail-induced apoptosis. Cancer Res 2001;61:1138-1143.

26 Uno K, Inukai T, Kayagaki N, Goi K, Sato H, Nemoto A, Takahashi K, Kagami K, Yamaguchi N, Yagita H, Okumura K, Koyama-Okazaki T, Suzuki T, Sugita K, Nakazawa S: Tnf-related apoptosis-inducing ligand (trail) frequently induces apoptosis in philadelphia chromosome-positive leukemia cells. Blood 2003;101:3658-3667.

-27 Steinwede K, Henken S, Bohling J, Maus R, Ueberberg B, Brumshagen C, Brincks EL, Griffith TS, Welte T, Maus UA: Tnf-related apoptosis-inducing ligand (trail) exerts therapeutic efficacy for the treatment of pneumococcal pneumonia in mice. J Exp Med 2012;209:1937-1952.

28 Galligan L, Longley DB, McEwan M, Wilson TR, McLaughlin K, Johnston PG: Chemotherapy and trail-mediated colon cancer cell death: The roles of p53, trail receptors, and c-flip. Mol Cancer Ther 2005;4:2026-2036.

29 Insinga A, Monestiroli S, Ronzoni S, Gelmetti V, Marchesi F, Viale A, Altucci L, Nervi C, Minucci S, Pelicci PG: Inhibitors of histone deacetylases induce tumor-selective apoptosis through activation of the death receptor pathway. Nat Med 2005;11:71-76.

-30 Carafa V, Miceli M, Altucci L, Nebbioso A: Histone deacetylase inhibitors: A patent review (2009 - 2011). Expert Opin Ther Pat 2013;23:1-17.

31 Chopin V, Slomianny C, Hondermarck H, Le Bourhis X: Synergistic induction of apoptosis in breast cancer cells by cotreatment with butyrate and tnf-alpha, trail, or anti-fas agonist antibody involves enhancement of death receptors' signaling and requires p21(waf1). Exp Cell Res 2004;298:560-573.

-32 Xu SQ El-Deiry WS: P21(waf1/cip1) inhibits initiator caspase cleavage by trail death receptor dr4. Biochem Biophys Res Commun 2000;269:179-190.

-33 Vignot S, Faivre S, Aguirre D, Raymond E: Mtor-targeted therapy of cancer with rapamycin derivatives. Ann Oncol 2005;16:525-537. 